\title{
A Unified Model for Support Vector Machine and Support Vector Data Description
}

\author{
Trung Le, Dat Tran, Wanli Ma, and Dharmendra Sharma \\ Faculty of Information Sciences and Engineering \\ University of Canberra, ACT 2601, Australia
}

\begin{abstract}
Support vector machine (SVM) and support vector data description (SVDD) are the well-known kernel-based methods for pattern classification. SVM constructs an optimal hyperplane whereas SVDD constructs an optimal hypersphere to separate data between two classes. SVM and SVDD have been compared in pattern classification experiments however there is no theoretical work on comparison between these methods. This paper presents a new theoretical model to unify SVM and SVDD. The proposed model constructs two optimal points to generate a general decision boundary which can be transformed to hyperplane for SVM or hypersphere for SVDD.

Index Terms-Novelty detection, one-class classification, support vector data description, and spherically shaped boundary.
\end{abstract}

\section{INTRODUCTION}

In pattern classification, a family of functions $f(x, \alpha), \alpha \in$ $\wedge$, is used to determine decision boundary. With a given family of function $(f(x, \alpha), \alpha \in \wedge)$, the best parameter $\alpha_{0}$ in terms of minimising general risk should be nominated. This task is challenging since we always have insufficient data for learning, only empirical risk could be determined. The relevance between the empirical risk and general risk is exhibited in the following inequality [9][8]:

For all $\eta>0$, with probability greater than $1-\eta$, the following inequality holds true:

$$
R(\alpha) \leq R_{e m p}(\alpha)+\frac{B \varepsilon}{2}\left(1+\sqrt{1+\frac{4 R_{e m p}(\alpha)}{B \varepsilon}}\right)
$$

where $\varepsilon=4 \frac{h\left(\ln \frac{2 n}{h}+1\right)-\ln \eta}{n}, B=1$ for pattern classification problem, $h$ is Vapnik-Cervonenkis dimension (VC-dimension) of the function family $f(x, \alpha), \alpha \in \wedge, n$ is the size of training set, and $R_{e m p}(\alpha) \operatorname{and} R(\alpha)$ are the empirical and general risks, respectively.

It is shown in (1) that there is a trade-off between $R_{e m p}(\alpha)$ and second summand. When the size of training set increases, $R_{e m p}(\alpha)$ increases but the second summand decreases and vice versa. Therefore, to minimize $R(\alpha)$ we target the function family $f(x, \alpha)$ and learning principles such that both the empirical risk $R_{e m p}(\alpha)$ and second summand are as small as possible.

There are two well-known learning principles which are Empirical Risk Minimization (ERM) and Structural Risk Minimization (SRM)[10]. Maximum Likelihood method (ML) [3] is a typical example that conforms to the ERM principle. In the ERM principle, the best parameter is learned from a given training set so that the empirical risk is minimized. Under some circumstances, the second summand in (1) is also minimized and we can see that the general risk is also small. On the other hand, a sequence of nested function families $S_{1} \subset S_{2} \subset S_{3} \subset \ldots \subset S_{k} \subset \ldots$ is constructed such that $S^{*}=\bigcup_{k} S_{k}$ is everywhere dense in $S$ where $S$ is the function family $f(x, \alpha), \alpha \in \wedge$. The SRM principle suggests that for a given training set, we choose the element of structure $S_{k}$, where $k=k(n)$ and choose a particular function from $S_{k}$ for which the general risk in (1) is minimized.

Support Vector Machine (SVM) [1], [2], [9] is a well-known kernel-based method in pattern classification. It is used to deal with the balanced datasets. An optimal hyperplane is constructed in feature space so that the margin, distance from the closest point to hyperplane, is maximized. The theoretical background of SVM is enforced by the following inequality:

$$
h \leq \min \left\{\left[\frac{R^{2}}{\Delta}\right], d\right\}+1
$$

where $h$ is VC-dimension of $\triangle$-margin separating hyperplane family, $\triangle$ is a separating margin, $[x]$ returns integer part of $\mathrm{x}, R$ is radius of the smallest hypersphere that can cover the training set. The objective function of SVM $\left(\frac{1}{2}\|w\|^{2}+C \sum_{i=1}^{n} \xi_{i}\right)$ involves maximization of margin and minimization of empirical risk $\left(R_{e m p}(\alpha)\right)$. According to (2), maximization of margin ensures the minimal VC-dimension $h$ and minimal second summand in (1) as well. Therefore, by referring to (1) the general risk must be minimized.

Support Vector Data Description (SVDD) [6] is specified for the imbalanced datasets. It aims at conducting an optimal hypersphere in feature space so that this hypersphere can include all normal data and exclude all abnormal data . The decision boundary is mainly learned from the normal (major) data whereas the abnormal (minor) data commits to refine the decision boundary [4]. There are some variations of SVDD. In Small Sphere Large Margin (SSLM) [11], an optimal hypersphere is appointed to include all normal data and exclude all abnormal data. The margin, distance between the closest abnormal data to surface of the optimal hypersphere, is maximized to increase the True Negative rate (TN\%). However, since the optimal hypersphere is too close to normal data, the True Positive rate (TP\%) can be decreased as a consequence. To deal with this side effect, an Optimal Sphere Two Large Margins (SS2LM) [7] targets building an 
optimal hypersphere with two proportional large margins. The ratio between two margins can be adjusted to balance TN\% and $\mathrm{TP} \%$.

Although SVM and SVDD are the well-known kernel-based methods in pattern classification to deal with different types of datasets and have similar learning frameworks, current studies have not developed a general framework that can unify these methods. In this paper, we introduce a new learning method that can be considered as a combination of SVM and SVDD. The proposed method constructs two optimal points as a general decision boundary which can be transformed to hyperplane for SVM or hypersphere for SVDD.

\section{Support Vector Machine}

In SVM [1], [2], [9], an optimal hyperplane is built up so that the margin, distance from the closest point to hyperplane, is maximized. The optimization problem (OP) is of the following form:

$$
\min _{w, b} \frac{1}{2}\|w\|^{2}+C \sum_{i=1}^{n} \xi_{i}
$$

subjects to

$$
\begin{aligned}
& y_{i}\left(w^{T} \phi\left(x_{i}\right)+b\right) \geq 1-\xi_{i}, i=1, \ldots, n \\
& \xi_{i} \geq 0, \quad i=1, \ldots, n
\end{aligned}
$$

where $C$ is constant, $\xi=\left[\xi_{i}\right]_{i=1, \ldots, n}$ is vector of slack variables, $\phi($.$) is a transformation from input space to feature$ space.

For classifying an unknown data point $x$, the following decision function is used: $f(x)=\operatorname{sign}\left(w^{T} \phi(x)+b\right)$.

\section{Support Vector Data Description}

SVDD [6] aims at determining an optimal hypersphere to include all normal data points while excluding all abnormal data points. The optimization problem is as follows:

$$
\min _{R, c, \xi} R^{2}+C \sum_{i=1}^{n} \xi_{i}
$$

subject to

$$
\begin{aligned}
& \left\|\phi\left(x_{i}\right)-c\right\|^{2} \leq R^{2}+\xi_{i} \quad i=1, \ldots, m \\
& \left\|\phi\left(x_{i}\right)-c\right\|^{2} \geq R^{2}-\xi_{i} \quad i=m+1, \ldots, n \\
& \xi_{i} \geq 0, \quad i=1, \ldots, n
\end{aligned}
$$

where $R$ is radius of the hypersphere, $C$ is constant, $\xi=$ $\left[\xi_{i}\right]_{i=1, \ldots, n}$ is vector of slack variables, $m$ is the number of normal data points, $\phi($.$) is a transformation from input space$ to feature space, and $c$ is center of the hypersphere.

For classifying an unknown data point $x$, the following decision function is used: $f(x)=\operatorname{sign}\left(R^{2}-\|\phi(x)-c\|^{2}\right)$. The unknown data point $x$ is normal if $f(x)=+1$ or abnormal if $f(x)=-1$.

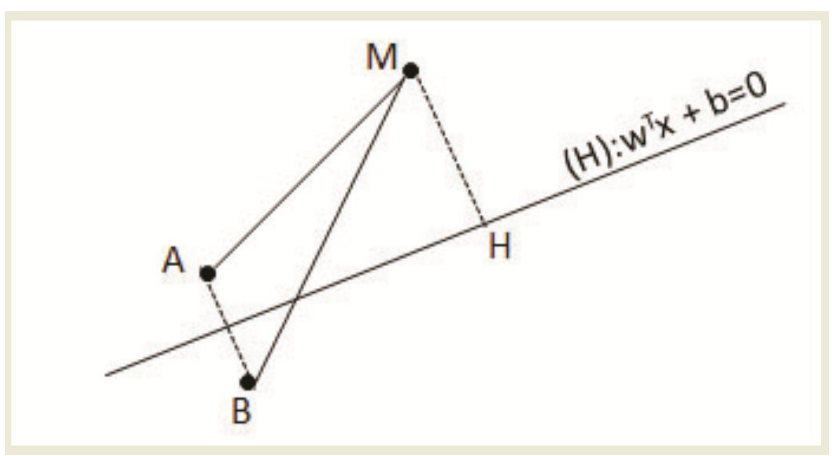

Fig. 1. They key lemma of new model.

\section{The Unified Model}

\section{A. Key Lemma}

This lemma is very important for derivation of new proposed model. It displays the relevance between the difference of square distance to two points and the margin in the affine space $R^{d}$.

Key Lemma

Let $M, A, B$ be the points in the affine space $R^{d}$ respectively. Let $(H): w^{T} x+b=0$ be the mid-perpendicular hyperplane of segment $A B$. The following equality holds:

$$
M A^{2}-M B^{2}=2 \operatorname{dis}(M, \mathrm{H}) \cdot A B \cdot \operatorname{sign}(B, H) \cdot \operatorname{sign}(M, H)
$$

where $\operatorname{dis}(M, H)$ is distance from point $M$ to hyperplane $(H)$, $\operatorname{sign}(P, H)$ is the sign of value of substitution point $P$ to hyperplane $(H)$ or in the other hand $\operatorname{sign}\left(w^{T} P+b\right)$.

\section{Proof}

$$
\begin{aligned}
& M A^{2}-M B^{2}=\overrightarrow{M A}^{2}-\overrightarrow{M B}^{2}=(\overrightarrow{M A}-\overrightarrow{M B})(\overrightarrow{M A}+\overrightarrow{M B}) \\
& =2 \overrightarrow{B A} \cdot \overrightarrow{M I}=2 \overrightarrow{B A}(\overrightarrow{M H}+\overrightarrow{H I})
\end{aligned}
$$

where $I$ is the midpoint of segment $A B, H$ is projected point of $M$ to hyperplane $(H)$ as in Figure (1).

Since $\overrightarrow{H I}$ is orthogonal to $\overrightarrow{B A}$ and $\overrightarrow{M H}$ is parallel to $\overrightarrow{B A}$, we have:

$$
\begin{aligned}
& M A^{2}-M B^{2}=2 \overrightarrow{B A} \cdot \overrightarrow{M H} \\
& =2 B A \cdot M H \cdot \operatorname{sign}(B, H) \cdot \operatorname{sign}(M, H) \\
& =2 B A \cdot \operatorname{dis}(M, H) \cdot \operatorname{sign}(B, H) \cdot \operatorname{sign}(M, H)
\end{aligned}
$$

\section{B. The Idea of the Proposed Model}

We are going to look at the original support vector machine in the different view to derive the new model. The key idea of original SVM is to specify the optimal hyperplane so that the margin, the distance from the closest point of training set to hyperplane, is maximized. It can be restated in the another form as bellows:

$$
\max _{w, b} \gamma
$$

subject to

$$
\operatorname{dis}(M, H) \geq \gamma \text { for all } M \in \phi(X)
$$


where $X$ is training set, $\phi(X)$ is image of $X$ through the transformation $\phi(),$.$M is a vector of training set, (H)$ : $w^{T} \phi(x)+b=0$ is a hyperplane in feature space, and $\operatorname{dis}(M, H)$ is distance from vector $\mathrm{M}$ to hyperplane $(H)$.

By referring to proposition key lemma, it is able to transform the above optimization problem to the another equivalent form as follows:

$$
\max _{A, B} \frac{\gamma}{A B}
$$

subject to

$$
\left(M A^{2}-M B^{2}\right) y_{M} \geq \gamma \text { for all } M \in \phi(\mathrm{X})
$$

where $y_{M}$ is the label of vector(point) $M, A$ and $B$ are two vectors (points) in feature space, and mid-perpendicular hyperplane of segment $A B$ will form the optimal hyperplane.

We will convert the above optimization problem to the more concrete form. It is pleased to note that the objective function in (11) is margin, distance from the closest point of training set to the mid-perpendicular hyperplane of segment $A B$ and this margin still keeps invariant when stretching or shrinking segment $A B$ as long as the mid-perpendicular hyperplane of segment $A B$ is stationary. Therefore, without loss of generality we can assume that:

$$
\min _{M}\left(M A^{2}-M B^{2}\right) y_{M}=1
$$

Consequently, we achieve new equivalent optimization problem:

$$
\max _{A, B} \frac{1}{A B}
$$

subject to

$$
\left(M A^{2}-M B^{2}\right) y_{M} \geq 1 \text { for all } M \in \phi(\mathrm{X})
$$

or the another equivalent form

$$
\min _{A, B} A B^{2}
$$

subject to

$$
\left(M A^{2}-M B^{2}\right) y_{M} \geq 1 \text { for all } M \in \phi(\mathrm{X})
$$

Similar to SVM, we have a chance to extend the problem by applying the slack variables as follows:

$$
\min _{A, B} A B^{2}+C \sum_{i=1}^{n} \xi_{i}
$$

subject to

$$
\begin{aligned}
& \left(M_{i} A^{2}-M_{i} B^{2}\right) y_{i} \geq 1-\xi_{i} \text { for all } M_{i} \in \phi(\mathrm{X}) \\
& \xi_{i} \geq 0, i=1, \ldots, n
\end{aligned}
$$

\section{Formulation}

To resolve the new optimization problem, let us denote the coordinate of points $A, B, M$ in feature space by $a, b, \phi(x)$ respectively. We come up with new OP as follows:

$$
\min _{a, b, \xi}\|b-a\|^{2}+C \sum_{i=1}^{n} \xi_{i}
$$

subject to

$$
\begin{aligned}
& \left(\left\|\phi\left(x_{i}\right)-a\right\|^{2}-\left\|\phi\left(x_{i}\right)-b\right\|^{2}\right) y_{i} \geq 1-\xi_{i} \\
& \xi_{i} \geq 0, i=1, \ldots, n
\end{aligned}
$$

where $\phi$ is a transformation from the input space to the feature space.

We generalize the above optimization problem by introducing the new parameter called "curving degree" $k$. As explained later, this parameter governs curving degree of decision boundary. When $k=1$, it is completely straight. Moreover, when $k \neq 1$, it becomes spherical form with varied-curving degree. We obtain new optimization problem as follows:

$$
\min _{a, b, \xi}\|b-a\|^{2}+C \sum_{i=1}^{n} \xi_{i}
$$

subject to

$$
\begin{aligned}
& \left(\left\|\phi\left(x_{i}\right)-a\right\|^{2}-k\left\|\phi\left(x_{i}\right)-b\right\|^{2}\right) y_{i} \geq 1-\xi_{i}, \mathrm{i}=1, \ldots, \mathrm{n} \\
& \xi_{i} \geq 0, i=1, \ldots, n
\end{aligned}
$$

\section{Solution}

To derive the new optimization problem, we refer to the Karush-Kuhn-Tucker (KKT) theorem. The Lagrange function is of:

$$
\begin{aligned}
& L(a, b, \xi, \alpha, \beta)=\|b-a\|^{2}+C \sum_{i=1}^{n} \xi_{i}- \\
& \sum_{i=1}^{n} \alpha_{i}\left(\left(\left\|\phi\left(x_{i}\right)-a\right\|^{2}-k\left\|\phi\left(x_{i}\right)-b\right\|^{2}\right) y_{i}-1+\xi_{i}\right) \\
& -\sum_{i=1}^{n} \beta_{i} \xi_{i}
\end{aligned}
$$

Setting the derivatives to 0 , we obtain

$$
\begin{gathered}
\frac{\delta L}{\delta a}=0 \Rightarrow a-b=\sum_{i=1}^{n} \alpha_{i} y_{i}\left(a-\phi\left(x_{i}\right)\right) \\
\frac{\delta L}{\delta b}=0 \Rightarrow b-a=k \sum_{i=1}^{n} \alpha_{i} y_{i}\left(\phi\left(x_{i}\right)-b\right) \\
\frac{\partial L}{\partial \xi_{i}}=0 \Rightarrow \alpha_{i}+\beta_{i}=C
\end{gathered}
$$

Timing (25) to $k$ and adding to (26), we have:

$$
(k-1)(a-b)=k \sum_{i=1}^{n} \alpha_{i} y_{i}(a-b)
$$


Since $a-b \neq 0$. Hence, we gain: $\sum_{i=1}^{n} \alpha_{i} y_{i}=\frac{k-1}{k}$. Substituting (28) with (25), we have $a-k b=$ $-k \sum_{i=1}^{n} \alpha_{i} y_{i} \phi\left(x_{i}\right)$.

Substituting back to (24), we achieve the dual form:

$$
\begin{aligned}
& L(a, b, \xi, \alpha, \beta)=\|b-a\|^{2} \\
& -\sum_{i=1}^{n} \alpha_{i}\left(\left(\left\|\phi\left(x_{i}\right)-a\right\|^{2}-k\left\|\phi\left(x_{i}\right)-b\right\|^{2}\right) y_{i}-1\right) \\
& =\|b-a\|^{2}+(k-1) \sum_{i=1}^{n} y_{i} \alpha_{i} K\left(x_{i}, x_{i}\right) \\
& +2(a-k b) \sum_{i=1}^{n} \alpha_{i} y_{i} \phi\left(x_{i}\right)-\left(\|a\|^{2}-k\|b\|^{2}\right) \sum_{i=1}^{n} \alpha_{i} y_{i} \\
& +\sum_{i=1}^{n} \alpha_{i} \\
& =\|b-a\|^{2}-\frac{2}{k}\|a-k b\|^{2}-\frac{(k-1)}{k}\left(\|a\|^{2}-k\|b\|^{2}\right) \\
& +\sum_{i=1}^{n} \alpha_{i}+(k-1) \sum_{i=1}^{n} y_{i} \alpha_{i} K\left(x_{i}, x_{i}\right) \\
& =\frac{-1}{k}\|a-k b\|^{2}+\sum_{i=1}^{n} \alpha_{i}+(k-1) \sum_{i=1}^{n} y_{i} \alpha_{i} K\left(x_{i}, x_{i}\right) \\
& =-k\left\|\sum_{i=1}^{n} \alpha_{i} y_{i} \phi\left(x_{i}\right)\right\|^{2}+\sum_{i=1}^{n}\left((k-1) y_{i} K\left(x_{i}, x_{i}\right)+1\right) \alpha_{i}
\end{aligned}
$$

Therefore, we need to deal with the below optimization problem:

$$
\begin{aligned}
\min _{\alpha} & \left(k \sum_{i=1}^{n} \sum_{j=1}^{n} y_{i} y_{j} K\left(x_{i}, x_{j}\right) \alpha_{i} \alpha_{j}\right. \\
& \left.-\sum_{i=1}^{n}\left((k-1) y_{i} K\left(x_{i}, x_{i}\right)+1\right) \alpha_{i}\right)
\end{aligned}
$$

subject to

$$
\sum_{i=1}^{n} \alpha_{i} y_{i}=\frac{k-1}{k} ; 0 \leq \alpha_{i} \leq C, i=1, \ldots, n
$$

\section{E. Support Vectors}

We call $0<\alpha_{i}<C$ by support vector. Let us denote $I$ by set of indices of all support vectors i.e. $I=$ $\left\{i: 0<\alpha_{i}<C ; 1 \leq i \leq n\right\}$.

By referring to condition of KKT theorem as in SVM and SVDD, we yield:

$$
\left(\left\|\phi\left(x_{i}\right)-a\right\|^{2}-k\left\|\phi\left(x_{i}\right)-b\right\|^{2}\right) y_{i}=1
$$

or $\left\|\phi\left(x_{i}\right)-a\right\|^{2}-k\left\|\phi\left(x_{i}\right)-b\right\|^{2}=y_{i}$ since $y_{i}= \pm 1$ It means that:

$$
\begin{aligned}
& (1-k) K\left(x_{i}, x_{i}\right)-2(a-k b) \phi\left(x_{i}\right)+\|a\|^{2}-k\|b\|^{2}=y_{i} \\
& (1-k) K\left(x_{i}, x_{i}\right)+2 \phi\left(x_{i}\right) k \sum_{j=1}^{n} \alpha_{j} y_{j} \phi\left(x_{j}\right)+d=y_{i} \\
& (1-k) K\left(x_{i}, x_{i}\right)+2 k \sum_{j=1}^{n} \alpha_{j} y_{j} K\left(x_{i}, x_{j}\right)+d=y_{i} \\
& d=y_{i}+(k-1) K\left(x_{i}, x_{i}\right)-2 k \sum_{j=1}^{n} \alpha_{j} y_{j} K\left(x_{i}, x_{j}\right)
\end{aligned}
$$

where $d=\|a\|^{2}-k\|b\|^{2}$.

In practice, we calculate $d=\|a\|^{2}-k\|b\|^{2}$ by taking average right hand sides of (33) for all support vectors as follows:

$$
\begin{aligned}
& \|a\|^{2}-k\|b\|^{2}= \\
& \frac{1}{|I|} \sum_{i \in I}\left(y_{i}+(k-1) K\left(x_{i}, x_{i}\right)-2 k \sum_{j=1}^{n} \alpha_{j} y_{j} K\left(x_{i}, x_{j}\right)\right)
\end{aligned}
$$

\section{F. Decision Function}

For classifying an unknown data point $x$, the following decision function is used:

$$
\begin{aligned}
& f(x)=\operatorname{sign}\left(\|\phi(x)-a\|^{2}-k\|\phi(x)-b\|^{2}\right) \\
& =\operatorname{sign}\left((1-k) K(x, x)-2(a-k b) \phi\left(x_{i}\right)+\|a\|^{2}-k\|b\|^{2}\right) \\
& =\operatorname{sign}\left((1-k) K(x, x)+2 \phi(x) k \sum_{j=1}^{n} \alpha_{j} y_{j} \phi\left(x_{j}\right)+d\right) \\
& =\operatorname{sign}\left((1-k) K(x, x)+2 k \sum_{j=1}^{n} \alpha_{j} y_{j} K\left(x, x_{j}\right)+d\right)
\end{aligned}
$$

where $d$ can be computed as in (34).

\section{G. Analysis of the Proposed Model}

The equation's decision boundary is of the following form:

$$
\|\phi(x)-a\|^{2}-k\|\phi(x)-b\|^{2}=0
$$

To anayze the above equation, the following lemmas are necessary.

Lemma 1

Let $A, B$ be two distinct points in the affine space $R^{d}$. Construct the fixed point $\mathrm{P}$ such that $\overrightarrow{P A}=k \overrightarrow{P B}(k \neq 1)$. Then for all points $M$, the following equality holds:

$$
\begin{aligned}
& M A^{2}-k M B^{2}= \\
& (1-k) M P^{2}+P A^{2}-k P B^{2}
\end{aligned}
$$

\section{Proof}

$$
\begin{aligned}
& M A^{2}-k M B^{2}=\overrightarrow{M A}^{2}-k \overrightarrow{M B}^{2} \\
& =(\overrightarrow{M P}+\overrightarrow{P A})^{2}-k(\overrightarrow{M P}+\overrightarrow{P B}) \\
& =(1-k) \overrightarrow{M P}^{2}-2 \overrightarrow{M P}(\overrightarrow{P A}-k \overrightarrow{P B})+\overrightarrow{P A}^{2}-k \overrightarrow{P B}^{2} \\
& =(1-k) M P^{2}+P A^{2}-k P B^{2}
\end{aligned}
$$

\section{Lemma 2}

Let $A, B$ be two distinct points in the affine space $R^{d}$. The trajectory of points $\mathrm{M}$ enforced by equation $M A^{2}-k M B^{2}=$ 0 is one of the following forms:

i) If $k=1$ then it is a hyperplane that goes through the midpoint of $A B$ and perpendicular to $A B$.

ii) If $k \neq 1$ then it is a hypersphere with center $\mathrm{P}$ where $\overrightarrow{P A}=k \overrightarrow{P B}$.

\section{Proof}

i) If $k=1$ then we have $M A=M B$. It means that the trajectory of points $\mathrm{M}$ is the mid-perpendicular hyperplane of 
segment $\mathrm{AB}$.

ii) If $k \neq 1$ then by referring to lemma 1, we have:

$$
\begin{aligned}
& 0=M A^{2}-k M B^{2} \\
& =(1-k) M P^{2}+P A^{2}-k P B^{2} \\
& =(1-k) M P^{2}+\frac{k^{2}}{(k-1)^{2}} A B^{2}-\frac{k}{(k-1)^{2}} A B^{2} \\
& =(1-k) M P^{2}+\frac{k}{k-1} A B^{2}
\end{aligned}
$$

It implies that $M P=\frac{\sqrt{k}}{|k-1|} A B$ or trajectory of all points $\mathrm{M}$ is a hypersphere with center $\mathrm{P}$.

The lemma 2 reveals that the form of decision boundary ruled by (36) is either hyperplane or hypersphere according to parameter $\mathrm{k}$. This parameter also governs the curving degree of decision boundary. Hence it is called curving degree parameter. Moreover, the range of expression of new model is wider than both SVM and SVDD. Therefore, it appears that new model is easier to fit the real datasets than both SVM and SVDD.

\section{USVM IN COMPARISON TO SVM AND SVDD}

\section{A. Theoretical Comparison}

We compare our model Unified Support Vector Machine (UCSVM) with SVM and SVDD. The quadratic optimization behind USVM, SVM and SVDD are respectively shown as follows:

USVM:

$$
\begin{aligned}
\min _{\alpha} & \left(k \sum_{i=1}^{n} \sum_{j=1}^{n} y_{i} y_{j} K\left(x_{i}, x_{j}\right) \alpha_{i} \alpha_{j}\right. \\
- & \left.\sum_{i=1}^{n}\left((k-1) y_{i} K\left(x_{i}, x_{i}\right)+1\right) \alpha_{i}\right)
\end{aligned}
$$

subject to

$$
\sum_{i=1}^{n} \alpha_{i} y_{i}=\frac{k-1}{k} ; 0 \leq \alpha_{i} \leq C, i=1, \ldots, n
$$

SVM [1], [2], [9]:

$$
\min _{\alpha}\left(\frac{1}{2} \sum_{i=1}^{n} \sum_{j=1}^{n} y_{i} y_{j} K\left(x_{i}, x_{j}\right) \alpha_{i} \alpha_{j}-\sum_{i=1}^{n} \alpha_{i}\right)
$$

subject to

$$
\sum_{i=1}^{n} \alpha_{i} y_{i}=0 ; 0 \leq \alpha_{i} \leq C, i=1, \ldots, n
$$

SVDD [6]:

$$
\begin{aligned}
& \min _{\alpha}\left(\sum_{i=1}^{n} \sum_{j=1}^{n} y_{i} y_{j} K\left(x_{i}, x_{j}\right) \alpha_{i} \alpha_{j}-\right. \\
& \left.\sum_{i=1}^{n} y_{i} K\left(x_{i}, x_{i}\right) \alpha_{i}\right)
\end{aligned}
$$

subject to

$$
\sum_{i=1}^{n} \alpha_{i} y_{i}=1 ; 0 \leq \alpha_{i} \leq C, i=1, \ldots, n
$$

To see the relationship between USVM and SVM, let us replace $\alpha_{i}$ with $\frac{1}{2} \alpha_{i}$, and then let $k=1$. We achieve the same

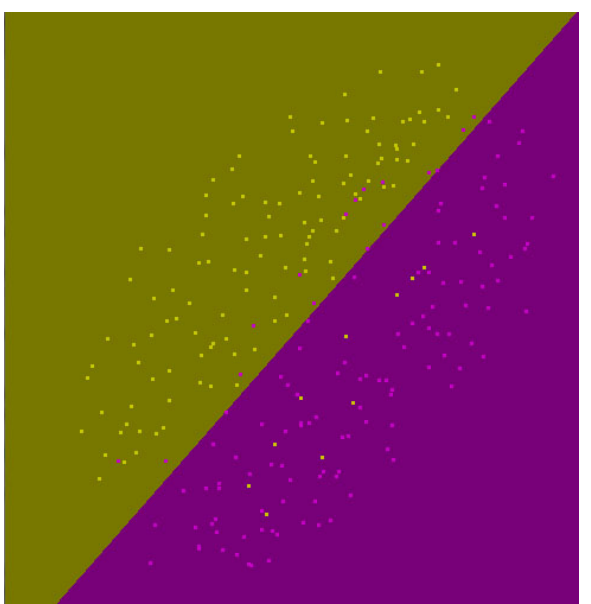

Fig. 2. SVM for first dataset

quadratic optimization problem as in SVM. It shows that SVM is a special case of USVM when $k=1$.

In (40), let us divide the objective function by $k$ and let $k$ approach $+\infty$. Amazingly, we obtain the same quadratic optimization problem as that in SVDD. It indicates that USVM can approximate SVDD when the "curving degree" $k$ approaches $+\infty$.

Therefore, USVM can be considered as an extension and unification of SVM and SVDD. As a result USVM can be applied to both binary classification and one-class classification problem.

The above remarks also help us in design of experiment. In fact, in our experiment parameter $k$ will be varied in two domains. The first domain is around 1 to approximate SVM. The second domain approaches $+\infty$ to approximate SVDD.

\section{B. Experimental Comparison}

To show that SVM and SVDD are two special cases of USVM as $k=1$ and $k$ approaches $+\infty$, respectively. We run USVM, SVM and SVDD on 2D synthetic datasets. To directly view picture of data in feature space, we employed linear kernel in this demonstration.

For the first synthetic dataset as seen in figures (2) and (3), the decision boundaries of SVM and USVM as $k=1$ are the same. In addition, in figure (4) when we vary $k$ in its domain, a wider class of decision boundary is provided. It is obvious that decision boundary as $k=0.1$ is better than that of SVM or USVM as $k=1$.

For the second synthetic dataset, we run both SVDD and USVM with $C=300$. As seen in figures (5), (6), and (7), when $k$ increases, USVM approaches SVDD.

\section{EXPERIMENTAL RESULTS}

\section{A. Experiment on 24 real datasets}

To persuasively show the performance of new proposed model, we establish experiment on 24 datasets as in Table 1. Most of them are two-class datasets and some of them are multi-class datasets. For each dataset, we select two classes 


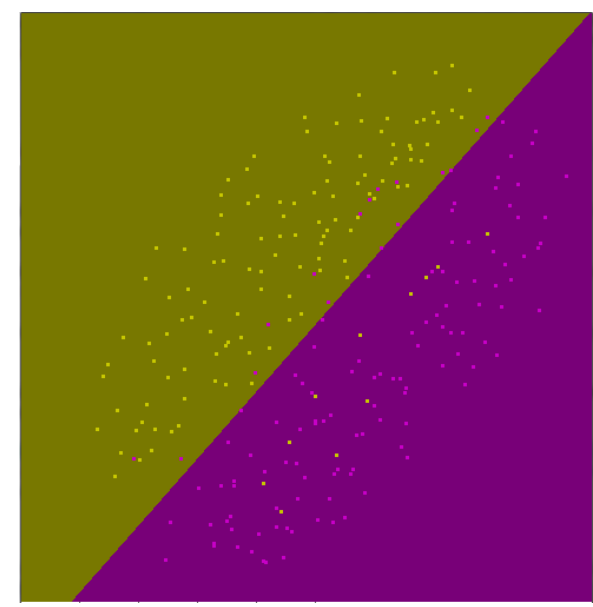

Fig. 3. USVM for first dataset $\mathrm{k}=1$

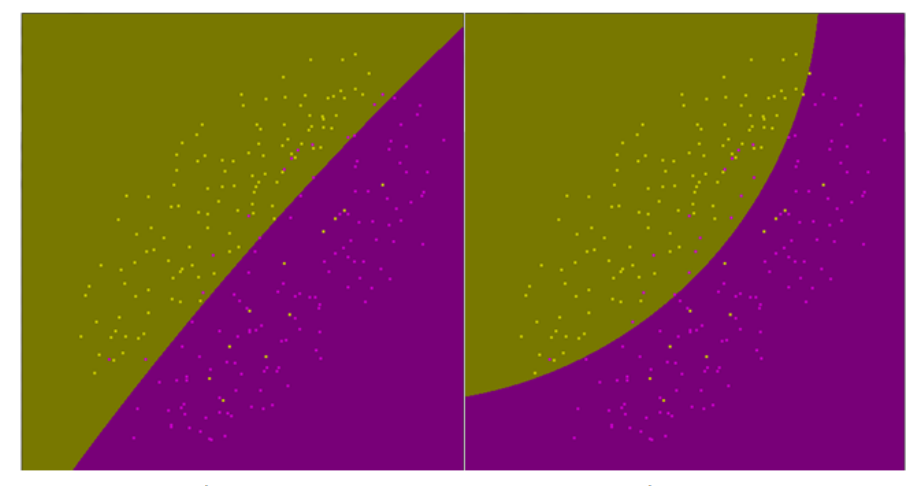

$k=0.1$

$k=10$

Fig. 4. USVM for first dataset when $\mathrm{k}$ varies

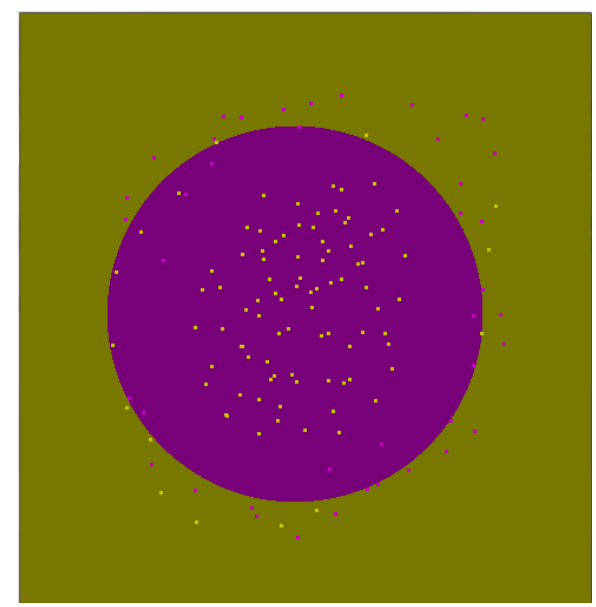

Fig. 5. SVDD for second dataset

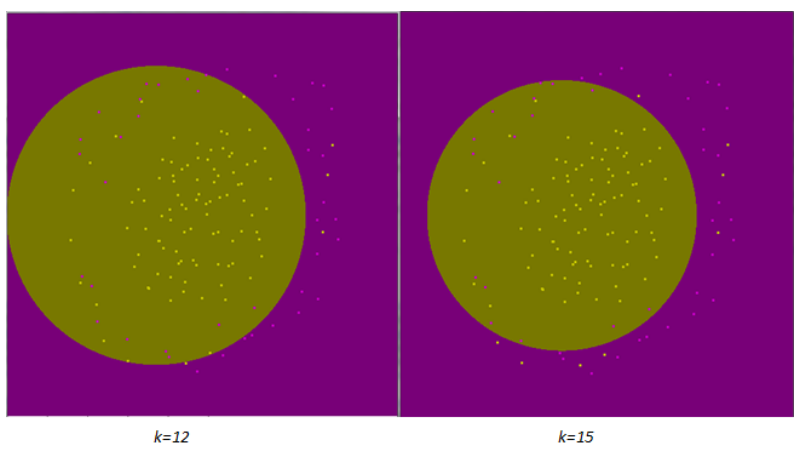

Fig. 6. USVM for second dataset as $\mathrm{k}=12$ and $\mathrm{k}=15$

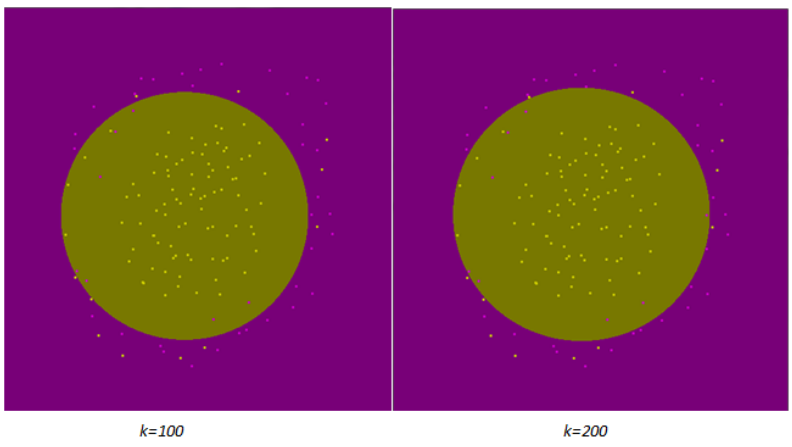

Fig. 7. USVM for second dataset as $\mathrm{k}=100$ and $\mathrm{k}=200$

and appoint one of them as normal class and the other one is abnormal. We run cross validation with five folds and calculate the average of accuracies on all folds to attain the cross validation accuracy.

The popular RBF Kernel $K\left(x, x^{\prime}\right)=e^{-\gamma\left\|x-x^{\prime}\right\|^{2}}$ is applied whereas the parameter $\gamma$ is varied in grid $\left\{2^{i}: i=2 j, j=\right.$ $-4, \ldots, 1\}$. The trade-off parameter $C$ is selected in grid $\left\{2^{i}\right.$ : $i=2 j, j=-4, \ldots, 1\}$. The curving degree $\mathrm{k}$ is searched in grid $\{0.6+0.2 i: i=0, \ldots, 5\} \cup\{5 i: i=2, \ldots, 10\}$. As mentioned in previous section, there are two domains for $\mathrm{k}$. The fist is to for SVM simulation and second is for SVDD simulation.

The experimental result is presented in Table 2 and the chart (8). It is shown that USVM outperforms SVM and SVDD on most datasets. It can be applied to both binary and one-class classification.

To indicate the relationship between curving parameter $k$ and accuracy, we visualize the plot of accuracy and $k$ in (9). The $\mathrm{x}$-axis indicates values of $k$. The $\mathrm{y}$-axis indicates the accuracies. It happens that the change of accuracy is quite stable along with $k$. It increases to its peak and then decreases. 
TABLE I

THE DETAILS OF EXPERIMENTAL DATASETS, \#NORMAL,\#ABNORMAL, AND D ARE NUMBER OF NORMAL, ABNORMAL DATA AND DIMENSION OF INPUT SPACE, RESPECTIVELY

\begin{tabular}{|c|c|c|l|}
\hline Datasets & \#normal & \#abnormal & $\# d$ \\
\hline Australian & 307 & 383 & 14 \\
\hline Arrhythmia & 237 & 183 & 278 \\
\hline Breast Cancer & 444 & 239 & 10 \\
\hline Biomed & 67 & 127 & 5 \\
\hline Astroparticle & 2000 & 1089 & 4 \\
\hline Wine & 59 & 71 & 13 \\
\hline Dna & 464 & 485 & 180 \\
\hline Fourclass & 307 & 555 & 2 \\
\hline Sonar & 97 & 111 & 67 \\
\hline Ionosphere & 255 & 126 & 34 \\
\hline Bioinformatics & 221 & 117 & 20 \\
\hline Diabetes & 500 & 268 & 8 \\
\hline Liver & 200 & 145 & 6 \\
\hline Letter & 549 & 567 & 16 \\
\hline Spectf & 254 & 95 & 44 \\
\hline SvmGuide3 & 296 & 947 & 22 \\
\hline Splice & 517 & 483 & 60 \\
\hline Vehicle & 212 & 217 & 18 \\
\hline Glass & 70 & 76 & 9 \\
\hline Delf Pump & 1124 & 376 & 64 \\
\hline German Number & 300 & 700 & 24 \\
\hline Heart & 164 & 139 & 13 \\
\hline Hepatitis & 32 & 123 & 19 \\
\hline Thyroid & 3679 & 93 & 21 \\
\hline & & &
\end{tabular}

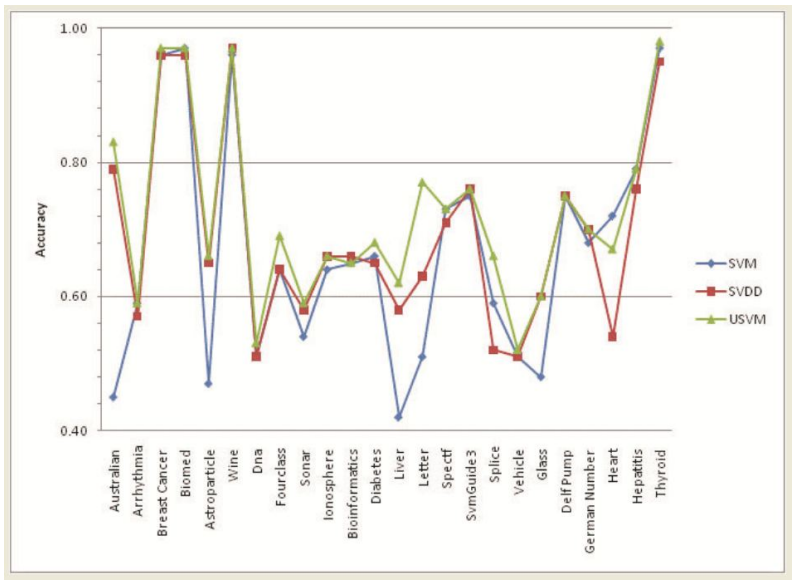

Fig. 8. Visualization of experimental result on 24 datasets.

TABLE II

THE EXPERIMENTAL RESULT SHOWS THE CROSS VALIDATION ACCURACIES FOR USVM, SVM AND SVDD ON 24 DATASETS

\begin{tabular}{|c|c|c|c|}
\hline Datasets & SVM & SVDD & USVM \\
\hline Australian & $45 \%$ & $79 \%$ & $83 \%$ \\
\hline Arrhythmia & $59 \%$ & $57 \%$ & $59 \%$ \\
\hline Breast Cancer & $96 \%$ & $96 \%$ & $97 \%$ \\
\hline Biomed & $97 \%$ & $96 \%$ & $97 \%$ \\
\hline Astroparticle & $47 \%$ & $65 \%$ & $66 \%$ \\
\hline Wine & $96 \%$ & $97 \%$ & $97 \%$ \\
\hline Dna & $51 \%$ & $51 \%$ & $53 \%$ \\
\hline Fourclass & $64 \%$ & $64 \%$ & $69 \%$ \\
\hline Sonar & $54 \%$ & $58 \%$ & $59 \%$ \\
\hline Ionosphere & $64 \%$ & $66 \%$ & $66 \%$ \\
\hline Bioinformatics & $65 \%$ & $66 \%$ & $65 \%$ \\
\hline Diabetes & $66 \%$ & $65 \%$ & $68 \%$ \\
\hline Liver & $42 \%$ & $58 \%$ & $62 \%$ \\
\hline Letter & $51 \%$ & $63 \%$ & $77 \%$ \\
\hline Spectf & $73 \%$ & $71 \%$ & $73 \%$ \\
\hline SvmGuide3 & $75 \%$ & $76 \%$ & $76 \%$ \\
\hline Splice & $59 \%$ & $52 \%$ & $66 \%$ \\
\hline Vehicle & $51 \%$ & $51 \%$ & $52 \%$ \\
\hline Glass & $48 \%$ & $60 \%$ & $60 \%$ \\
\hline Delf Pump & $75 \%$ & $75 \%$ & $75 \%$ \\
\hline German Number & $68 \%$ & $70 \%$ & $70 \%$ \\
\hline Heart & $72 \%$ & $54 \%$ & $67 \%$ \\
\hline Hepatitis & $79 \%$ & $76 \%$ & $79 \%$ \\
\hline Thyroid & $97 \%$ & $95 \%$ & $98 \%$ \\
\hline
\end{tabular}

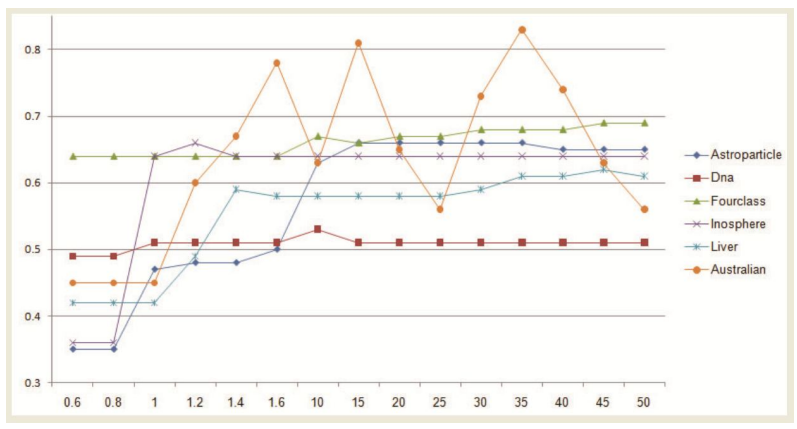

Fig. 9. The change of accuracy when $\mathrm{k}$ is varied on some datasets. 


\section{B. Experiment on INTERSPEECH 2010 Paralinguistic Chal- lenge Corpus}

To deeply evaluate our proposed method, we select the corpus of INTERSPEECH 2010 Paralinguistic Challenge [5]. This challenge includes three sub-challenges which are Gender Sub-Challenge, Age Sub-Challenge, and Affect SubChallenge. The participants of corpus collecting procedure were required to ring up the recording system six times. Each time they were reminded by an automated Interactive Voice Response system to repeat given utterances or produce free content. The speakers obtained individual prompt sheets containing the utterances and additional instructions. Between each session a break of one day was scheduled to ensure more variations of the voices. Each subject has six calls on a mobile phone alternating indoor and outdoor to obtain different recording environments.

In total, 47 hours of speech in 65364 single utterances of 954 speakers were populated. It is noted that not all volunteers completed all the six calls, and there were cases where some called more often than six times, resulting in different numbers of utterances per speaker. There were 770 speakers randomly selected as a Training partition (53076 utterances, 38.16 hours), which was further subdivided into Train (32527 utterances in 23.43 hours of speech of 471 speakers) and Develop (20549 utterances in 14.73 hours of speech of 299 speakers) partitions.

We mainly focus on Gender Sub-Challenge where it is required to classify utterances of children, female and male. The labels of utterances are $x, f$ and $m$ for children, female and male accordingly. The task of this challenge is to assign an arbitrary utterance to one of the labels $x, f$ or $m$. Therefore, it can be considered as multi-class classification problem. However, our purpose diverges from this requirement. We wish to prove that our model is able to not only simulate SVM and SVDD but also provides intermediate states which can produce better performance. With this purpose in mind, we design our own experiment. In fact, we run three binary classification problems for $\{x, f\},\{x, m\}$, and $\{f, m\}$.

As seen in our previous experiment, we applied RBF Kernel for SVM, SVDD and USVM. Setting up parameters was kept the same as those in our previous experiment except in USVM the parameter $k$ is searched in grid $\{0.98,0.985,0.99,0.995,1,1.05,1.1,1.15,1.2\}$.

The results of experiment are shown in Tables (3) and (4). We did not make comparison to competitors of this challenge. The reason is that our experiments were established for binary classification, not for multi-class classification as required in the challenge. Moreover, we have not been provided the testing set from this challenge. This led us to using the development set as the testing set. However, it seems that our results are promising in comparison to challenge baselines or other performances.
TABLE III

THE EXPERIMENTAL RESULT SHOWS THE CROSS VALIDATION ACCURACIES FOR USVM, SVM AND SVDD ON THE CORPUS OF INTERSPEECH 2010 PARALINGUISTIC CHALLENGE

\begin{tabular}{|c|c|c|l|}
\hline Datasets & SVM & SVDD & USVM \\
\hline$\{\mathrm{x}, \mathrm{f}\}$ & $93 \%$ & $63 \%$ & $94 \%$ \\
\hline$\{\mathrm{x}, \mathrm{m}\}$ & $88 \%$ & $58 \%$ & $97 \%$ \\
\hline$\{\mathrm{m}, \mathrm{f}\}$ & $79 \%$ & $68 \%$ & $86 \%$ \\
\hline
\end{tabular}

TABLE IV

THE EXPERIMENTAL RESULT SHOWS THE TESTING ACCURACIES FOR USVM, SVM AND SVDD ON THE CORPUS OF INTERSPEECH 2010 PARALINGUistic CHALLENGE

\begin{tabular}{|c|c|c|c|}
\hline Datasets & SVM & SVDD & USVM \\
\hline$\{\mathrm{x}, \mathrm{f}\}$ & $89 \%$ & $61 \%$ & $91 \%$ \\
\hline$\{\mathrm{x}, \mathrm{m}\}$ & $85 \%$ & $57 \%$ & $92 \%$ \\
\hline$\{\mathrm{m}, \mathrm{f}\}$ & $76 \%$ & $64 \%$ & $82 \%$ \\
\hline
\end{tabular}

\section{CONCLUSION}

We have proposed Unified SVM regarded as the general model for SVM and SVDD. We have proved that SVM and SVDD are special cases of the proposed model. The new model inherits the properties of SVDD and SVM, therefore it can be applied to both binary and one-class classification problem. It provides a wider range of applications in comparison to SVM and SVDD and can be applied to more real-world datasets than SVM and SVDD.

\section{REFERENCES}

[1] Burges, J. C. Christopher , "A Tutorial on Support Vector Machines for Pattern Recognition", Journal of Data Mining and Knowledge Discovery. vol. 2, pp. 121-167, 1998

[2] C. Cortes, and V. Vapnik, "Support-Vector Networks", Journal of Machine Learning. vol. 20, pp. 273-297, 1995

[3] A. Dempster, P.N. Laird, D. B. Rubin, "Maximum likelihood from incomplete data via the em algorithm", Journal of the Royal Statistical Society. vol. 39, pp. 1-38, 1977

[4] M. M. Moya, M. W. Koch, L. D. Hostetler, "One-class classifer networks for target recognition applications", Proc. of world congress on neural networks, 1991

[5] Schuller, B., Steidl, S., Batliner, A., Burkhardt, F., Devillers, L., Mller, C., and Narayanan, Shrikanth S., "The INTERSPEECH 2010 paralinguistic challenge", in INTERSPEECH, pp. 2794-2797, 2010

[6] D. M. Tax, R. P. Duin, "Support vector data description". Machine Learning. vol. 54, pp. 45-56, 2004

[7] Trung, L., Dat, T., Wanli, M., Dharmendra, S., "An optimal sphere and two large margins approach for novelty detection". Proc. IEEE World Congress on Computational Intelligence (WCCI), 2010

[8] Vapnik, V.: Estimation of Dependencies Based on Empirical Data. Springer-Verlag, New York(1982)

[9] Vapnik, V.: The Nature of Statistical Learning Theory. Springer-Verlag, New York(1995)

[10] Vapnik, V.: An overview of statistical learning theory. IEEE Transactions Neural Networks. 10, 988-999 (1999)

[11] Yu, M., Ye, J.:A small sphere and large margin approach for novelty detection using training data with outliers. IEEE Transaction on Pattern Analysis and Machine Intelligence. 31, 2088-2092 (2009) 